\title{
RESISTANCE CHARACTERISTICS OF SUBMERGED PROJECTILE WITH BOW VARIATION BASED ON HULL ENVELOPE EQUATION USING STEADY RANS SIMULATION
}

\author{
Yanuar $^{1 *}$, Ibadurrahman ${ }^{1}$, Syifa Alfiah Andini Putri ${ }^{1}$ \\ ${ }^{1}$ Department of Mechanical Engineering, Faculty of Engineering, Universitas Indonesia, Kampus UI \\ Depok, Depok 16424, Indonesia
}

(Received: July 2018 / Revised: October 2018 / Accepted: April 2019)

\begin{abstract}
This paper investigates the influence of the forebody of a projectile-shaped model without appendages and propulsion system in a submerged condition. The commercial steady RANS code, ANSYS Fluent, was used to conduct the simulations, and the forebody was varied based on the Hull Envelope equation. From the results, the model with $n f=2.75$ was the optimum design according to the bow efficiency coefficient. However, the model with a blunt form ( $n f=$ 1) produced the lowest drag because it had the least wet surface area. For models with high $n f$, a high accelerating flow led to a low-pressure condition after the impact of the fluid on the fore end. This soaring pressure difference caused a flow separation, and therefore the fullness of the forebody affected the fluid flow around the body: the alteration of pressure, the flow speed, and friction as the primary component of resistance.
\end{abstract}

Keywords: Bow shape; Hull envelope coefficient; Resistance characteristic; Steady RANS; Submerged projectile

\section{INTRODUCTION}

The resistance of a submerged object has a significant influence on its speed and acoustic signature, and hence its endurance. Viscous resistance is the only component of total drag on a deeply submerged object and is divided into two elements: skin-friction resistance and form (viscous pressure) resistance (Renilson, 2015). One way to reduce these resistances in the initial design stage is to consider the hull form of the vehicle in terms of its hydrodynamic aspect (Joubert, 2004).

Generally, the hull of a submerged object or submarine consists of three sections: the forebody, middle body and aft body (Burcher \& Rydill, 1994). The "Hull Envelope" formula, the first developed equation for a true teardrsop shape, is essential in the concept design stage (Jackson, 1992). Other designers have used different hull form equations for submerged objects or submarines, but they have still been associated with the teardrop shape (Kormilitsin \& Khalizev, 2001). The forebody of a submerged object determines the stagnation point (location and pressure) of the flow. Moreover, the bow shape has a significant influence on the behavior of fluid flow around and after it, thus affecting the effectiveness of sensitive equipment in it, e.g. acoustic or navigation equipment.

Following previous studies, an experiment on a submerged spheroid was conducted by Farell et al. (1973) in order to examine the influence of body form on the resistance components. Besides,

\footnotetext{
*Corresponding author's email yanuar@eng.ui.ac.id, Tel. +62-21-727-0032, Fax.+62-21-7270033

Permalink/DOI: https://dx.doi.org/10.14716/ijtech.v10i4.2156
} 
a wind-tunnel experiment on a high-speed underwater object reasonably demonstrated that an ellipsoidal nose profile could improve hydrodynamic performance (Suman et al., 2010). A windtunnel observation of a submersible vehicle also showed a typical flow separation in the symmetric bow of a submarine model with an angle-of-attack variation (Saeidinezhad et al., 2015).

The use of Computational Fluid Dynamics (CFD) has gained increasing favor in recent decades, during which time computing power and improvements in numerical algorithms have been improving significantly; Using CFD could straightforwardly construct a virtual towing tank or wind tunnel (Yang \& Löhner, 2003; Toxopeus, 2008; Fedor, 2009; Gross et al., 2011). The commercial CFD code ANSYS had been used to investigate an autonomous underwater vehicle and has resulted in a propeller race deduction (Rattanasiri et al., 2015). Another CFD approach showed that the elliptical submarine nose-shape was preferred for an initial design (Moonesun et al., 2016). Furthermore, Siswantara et al. (2016) have proven that the CFD method could be used for various digester designs to find that which is optimal in terms of slurry flow; they modelled in two dimensions to reduce the complexity of the calculations.

Investigation of a moving submerged object has focused mainly on achieving an optimum design by identifying the body form. Work using 2D steady-state flow RANS (Reynold Averaged NavierStokes) with variants of forebody shape and the speed range is well underway; the commercial CFD software ANSYS Fluent had been utilized in this study. The objective of this paper is to examine the characteristics of resistance and flow influenced by the bow form. The result may help designers at a concept design phase. The scope of the study focuses only on the forebody form of a projectile-like object in deeply submerged conditions, with the only drag components considered important being skin friction and viscous pressure resistance.

\section{PROBLEM DEFINITION}

\subsection{The Forebody}

The bow design aims to ensure the surrounding fluid flowed as to be possibly laminar over the forward sonar array or any sensitive equipment. The laminar flow is also to be extended as far as possible. In the axisymmetric bow form, the forebody is formed by revolving an ellipse about its major axis and can be described by the "Hull Envelope" equation:

$$
r_{x f}=\frac{D}{2}\left[1-\left(\frac{x_{f}}{L_{f}}\right)^{n_{f}}\right]^{\frac{1}{n_{f}}}
$$

where $r_{x f}$ is the radius at $x_{f}$ distance in the x-direction from the rearmost part of the forebody, as shown in Figure 1; $L_{f}$ is the length of the forebody; $D$ is the hull diameter; and $n_{f}$ designates a coefficient which defines the fullness of the forebody. If $n f$ equals 1 , the bow shape takes a conical form; if it equals 2 , the bow shape forms an ellipse.

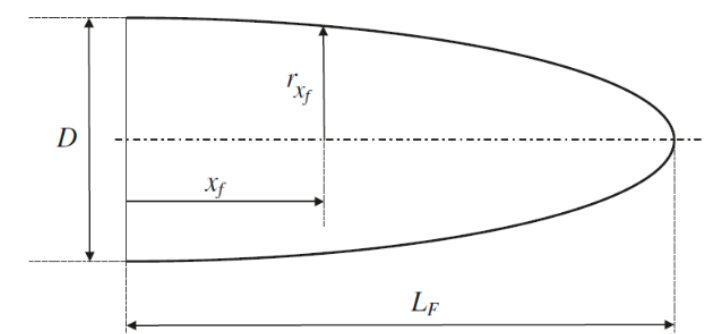

Figure 1 Schematic of the Forebody (Renilson, 2015) 


\subsection{Governing Equations}

Numerical simulations were performed using the commercial CFD software ANSYS Fluent. The flow around the vehicle was modelled using 2D incompressible flow; the steady-state RANS equations are:

Continuity

$$
\frac{\partial}{\partial x_{i}}\left(\overline{\rho u_{\imath}}\right)=0
$$

Momentum

$$
\frac{\partial}{\partial x_{j}}\left(\overline{\rho u_{l} u_{J}}\right)=-\frac{\overline{\partial p}}{\partial x_{i}}+\frac{\partial}{\partial x_{j}}\left[\mu\left(\frac{\overline{\partial u_{l}}}{\partial x_{i}}+\frac{\overline{\partial u_{j}}}{\partial x_{j}}-\frac{2}{3} \delta_{i j} \frac{\overline{\partial u_{l}}}{\partial x_{l}}\right)\right]+\frac{\partial}{\partial x_{j}}\left(-\overline{\rho u_{l}^{\prime} u_{J}^{\prime}}\right)
$$

where $\bar{\rho}$ is the mean density, $\bar{p}$ is the mean pressure, $\mu$ is the molecular viscosity and $\left(-\overline{\rho u^{\prime}{ }_{\imath} u_{J}^{\prime}}\right)$ identifies the Reynolds stresses. The challenge of acquiring a high quality solution was met by using the RANS equation to model the Reynold stress tensor to a satisfactory level, and was achieved by using a turbulence model. The $K-\varepsilon$ model was chosen for this study as it represents a good compromise between robustness, computational cost, and accuracy (ANSYS, 2009; Cao, 2016).

The finite volume method was employed to obtain a solution to the spatially governing equations. Coupling pressure and velocity fields were achieved using a SIMPLE technique. A second-order upwind scheme was used for the convection and a central-differencing scheme for the diffusion terms.

The turbulence model has restrictions on $y^{+}$at the wall. The principal of the wall function intends to bridge the viscous flow near a wall and link the solution variables at the wall-adjacent cells to the corresponding quantities at the wall. The non-dimensional wall parameter $y+$ is defined as:

$$
y^{+}=\frac{\Delta y}{v} \sqrt{\frac{\tau_{w}}{\rho}}
$$

where $\Delta y$ is the distance from the first computational node to the wall, and the subscript $w$ denotes the wall properties.

\section{NUMERICAL SETUP}

\subsection{Model Description}

The investigated object in this paper was a submerged axisymmetric body similar to a torpedo or bullet-shape object, or any small submersible vehicle without appendages. This study focuses on the resistance characteristics that are affected by the bow shape and examined 16 bow models: five of the general forebody for validation, and eleven of the $n f$-based forebody with reference to the "Hull Envelope" equation. A simplified 2D model was employed to prove the effectiveness of the CFD calculation for an axisymmetric form in a steady-state condition. All parts of the submerged body excluding the forebody (bow) were constant: the aft body was conical, the middle body was cylindrical, and the bow was varied in each model (see Figure 2).

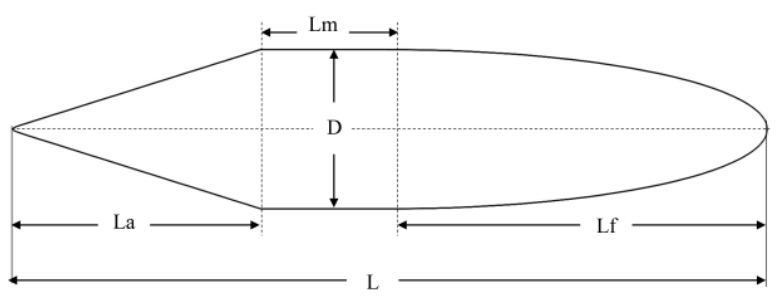

Figure 2 Hull parameter definition 
In order to provide similar hydrodynamic conditions, the model dimensions were kept in constant, as shown in Table 1. Although the bow was different in every model, the forebody length was relatively unchanged; this helped to evaluate the hydrodynamic aspect, specifically on the bow. However, each model had a different surface area as the forebody variations affected their shape, as demonstrated in Table 2. The surface area, as well as the bow arc, became more significant at a higher $n f$; the model with the highest $n f$ had a stub-like bow shape. The increment in $n f$ was equivalent to the model surface area as well as the bow arc.

Table 1 Specification of the models

\begin{tabular}{lccc}
\hline \multicolumn{1}{c}{ Property } & Symbol & Value & Units \\
\hline Length of the model & $L$ & 6 & $\mathrm{~m}$ \\
Forebody length & $L_{f}$ & 3 & $\mathrm{~m}$ \\
Middle body length & $L_{m}$ & 1 & $\mathrm{~m}$ \\
Aft body length & $L_{a}$ & 2 & $\mathrm{~m}$ \\
Length/ Diameter ratio & $L / D$ & 6 & - \\
Distance in x-direction & $x_{f}$ & 0.5 & $\mathrm{~m}$ \\
Test velocity & $v$ & $2-50$ & $\mathrm{knot}$ \\
\hline
\end{tabular}

Table 2 Specific description of the models

\begin{tabular}{clcc}
\hline Model & Bow shape & Bow area $\left(\mathrm{m}^{2}\right)$ & Bow arc $(\mathrm{m})$ \\
\hline 1 & conic & 1.500 & 6.083 \\
2 & ogive & 1.914 & 6.103 \\
3 & ellipse & 2.356 & 6.225 \\
4 & spherical & 2.893 & 6.571 \\
5 & stubbed & 2.996 & 6.914 \\
6 & $n f=1$ & 1.500 & 6.080 \\
7 & $n f=1.5$ & 2.107 & 6.212 \\
8 & $n f=2$ & 2.361 & 6.224 \\
9 & $n f=2.25$ & 2.490 & 6.295 \\
10 & $n f=2.5$ & 2.511 & 6.335 \\
11 & $n f=2.75$ & 2.618 & 6.343 \\
12 & $n f=3$ & 2.717 & 6.430 \\
13 & $n f=3.5$ & 2.841 & 6.576 \\
14 & $n f=4$ & 2.964 & 6.717 \\
15 & $n f=4.5$ & 2.972 & 6.762 \\
16 & $n f=5$ & 2.993 & 6.766 \\
\hline
\end{tabular}

The bow profile of all the models is shown in Figure 3. The five bow profiles (general form) were examined as a validation.
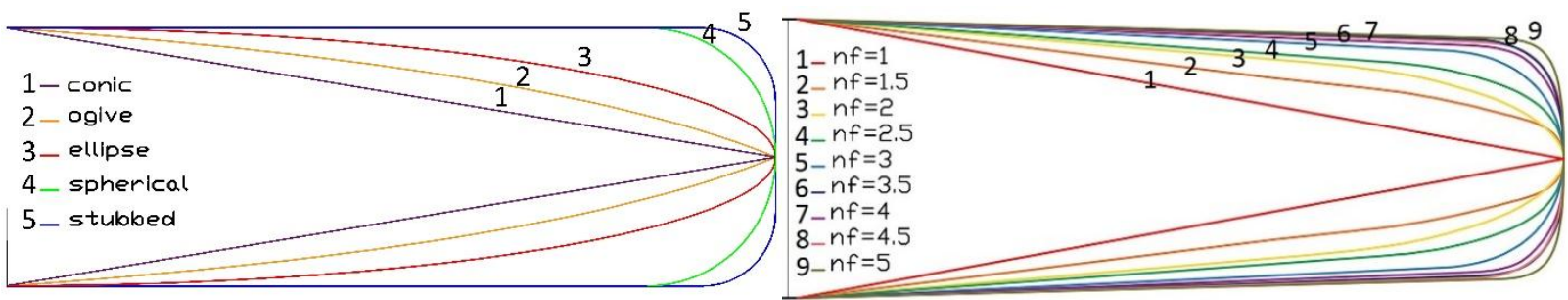

Figure 3 Bow profile of the general form (left) and the $n f$-based bow shape (right) 
The first model had a conic bow form, which is uncommon in any modern submarine or submerged object because it offers an insufficient amount of space for the acoustic equipment or other vital navigation components. Chen et al. (2012) had utilized the ogive-shaped and ellipseshaped bow on a novel underwater projectile by producing cavitation around its body. Spherical bow shape is a typical form used by modern submarines or torpedo-shaped projectiles (Kim et al., 2012). The stubbed form has low resistance in surface navigation but considerable resistance in a submerged mode of navigation (Renilson, 2015). The remaining profile of the forebody is the $n f$-based form, which was designed based on the "Hull Envelope Equation", varying from $n f$ $=1$ to $n f=5$.

\subsection{Boundary Conditions}

The boundary condition for the numerical simulation of the model is illustrated in Figure 4 . The inlet boundary was positioned at two body-lengths upstream, with an inflow velocity from 2 to 50 knots; the pressure-outlet condition was defined as four body-lengths downstream. A free slip wall boundary condition was applied to the remaining walls, one body-length away from the model. Moreover, a no-slip boundary condition was applied to the hull model.

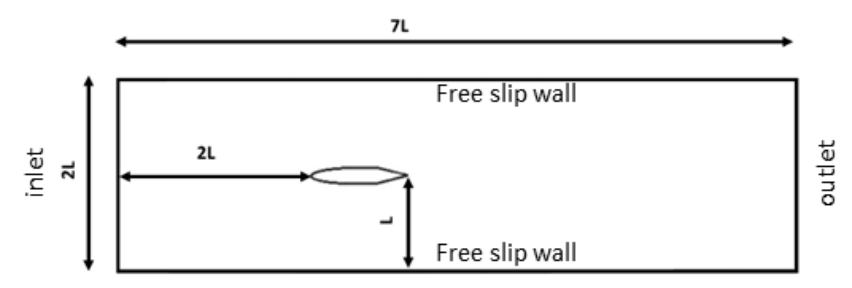

Figure 4 Boundary condition of the model

\subsection{Grid Generation}

The overall grid used in the simulation was a structured hexahedral mesh consisting of two parts: the domain of the flow and the model wall. The flow domain employed a mesh sizing with a face meshed treatment, while the model wall used a refinement treatment of the mesh with a refinement number equal to 3 .

Before conducting the numerical simulation, the sensitivity of the solution to the resolution of the grid was determined. Based on the base grid (rough), a series of refined grids was generated with a ratio of 0.5 in each step of refinement, as shown in Table 3. A medium mesh is depicted in Figure 5 (left) to visualize the grid appearance near the model.
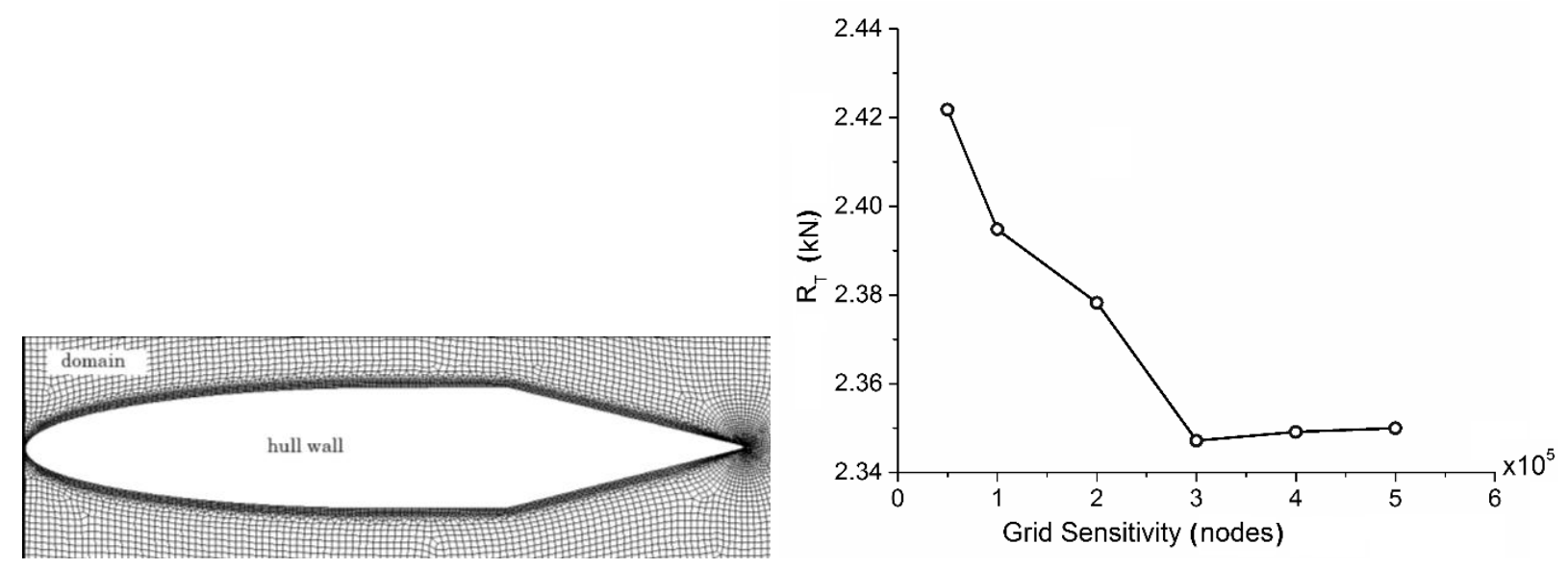

Figure 5 Mesh appearance (medium) close to the model (left) and grid independence (right)

The solution from the initial grid to the finest grid was compared to check any result variation due to the grid refinement. A mesh convergence test was conducted by focusing on longitudinal 
force; repeated convergence was assumed through normal residuals. Figure 5 (right) shows the grid independence. The solution changed significantly from the initial grid to the fair grade grid, whereas only minor differences were observed from the fair to the fine grid, as well as to the finest.

Moreover, $y^{+}$, as an important parameter related to the accuracy of the numerical prediction, varied from 2.83 (the finest mesh) to 5.01 (the coarsest mesh). Therefore, the finest grid was chosen for the simulation, with $y^{+}$at around 2.83 .

Table 3 Grid sensitivity

\begin{tabular}{lcc}
\hline \multicolumn{1}{c}{ Mesh } & Grid Quantity & $y^{+}$ \\
\hline Finest & $5 \times 10^{5}$ & 2.83 \\
Fine & $4 \times 10^{5}$ & 3.37 \\
Fair & $3 \times 10^{5}$ & 3.45 \\
Medium & $2 \times 10^{5}$ & 3.57 \\
Coarse & $1 \times 10^{5}$ & 4.60 \\
Rough & $5 \times 10^{4}$ & 5.01 \\
\hline
\end{tabular}

\section{RESULTS AND DISCUSSION}

\subsection{Validation}

The stability of all the equations was one parameter intended to achieve the convergence criteria; at this point, major flow features should be established. Figure 6 (left) depicts the residual history of the simulation. Generally, a decrease in residuals by three orders of magnitude indicates at least qualitative convergence (ANSYS, 2009). Furthermore, solution accuracy depends on the appropriateness of the physical models and boundary conditions.

Validation was performed by comparing the simulation results with related research. The benchmark data were produced from the results of the 3D model numerical simulation, with similar properties and a speed test of $10 \mathrm{~m} / \mathrm{s}$ (Moonesun et al., 2016). Figure 6 (right) depicts the validation of the models with the general form.

From the graph, neither set of total resistance coefficient $(C t)$ data showed any significant differences. A slight discrepancy between the two sets of data showed an average error of $11.1 \%$. The simulation results were slightly higher than those of the reference. However, both sets of data had a similar tendency. Even though this examination used a 2D model as a simplified tool for designers, the results are likely to be acceptable for initial estimation.
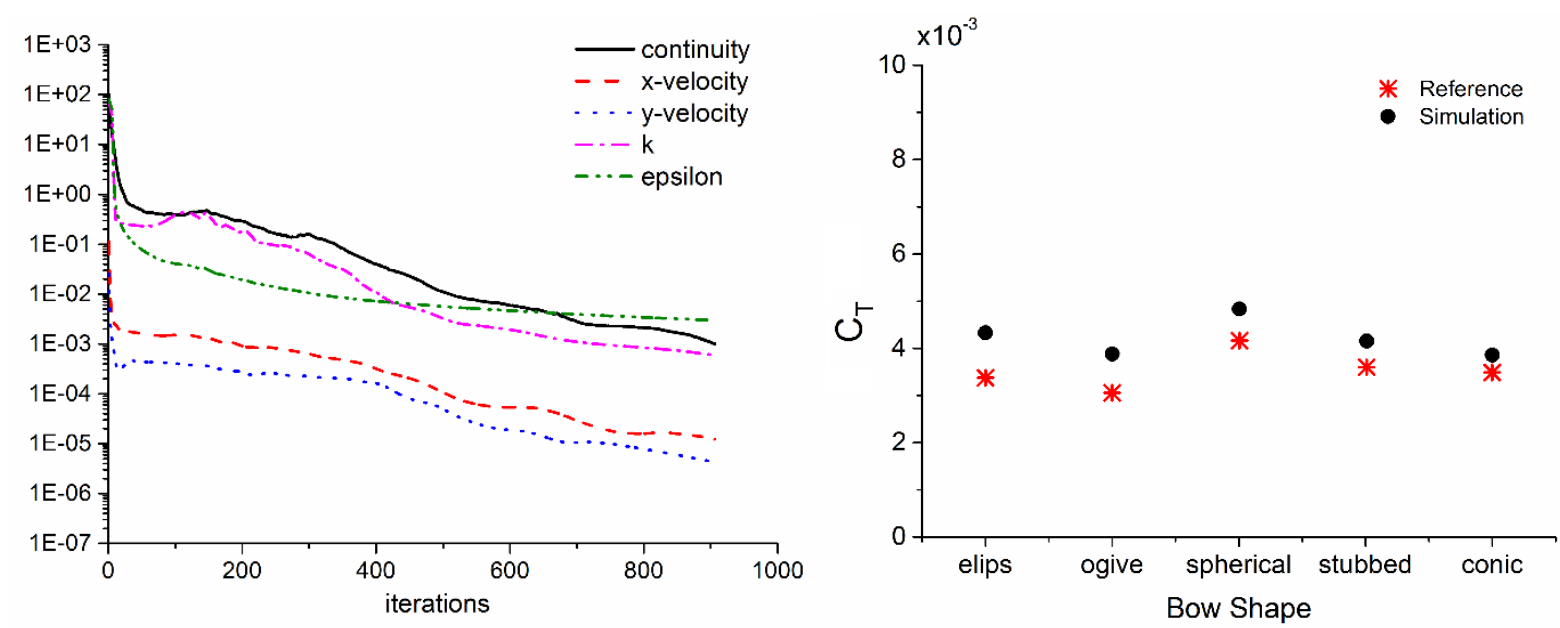

Figure 6 Residual history (left) and validation of models with the general forebody (right) 


\subsection{Numerical Simulation of Models with $\boldsymbol{n} \boldsymbol{f}$-based Bow Shape}

Designing a submerged object with forebody related to $n f$ directly impacts its wet surface area, and consequently affects its total resistance. The results were presented by its coefficient of resistance rather than magnitude because this adequately represents the resistance characteristics (Yanuar et al., 2017). Further, a non-dimensional parameter was used so that all the models could be appropriately compared.

Numerical simulations of the model with $n f$ from 1 to 5 were conducted using the finest quadrilateral nodes with 1000 iteration limit and the convergence criterion. The simulation was run with 25 different speed variations, from 2 to 50 knots. From the simulation, the total resistance coefficient $\left(C_{T}\right)$, skin friction resistance coefficient $\left(C_{F}\right)$, and viscous pressure resistance coefficient $\left(C_{P}\right)$ were examined. These parameters signalled specific resistance characteristics of the submerged model.

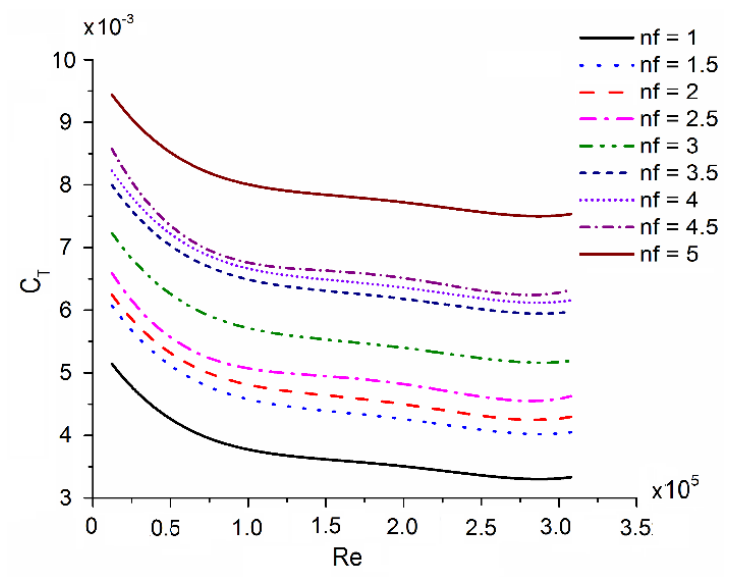

Figure 7 Total resistance coefficient of models with $n f$-based forebody

The total resistance coefficient describes a general aspect of the resistance characteristics of the model. Figure 7 shows the coefficient for each model based on its Reynold number. The $C_{T}$ on the range of $R e$ became more significant for models with larger $n f$; a higher wet surface area produced a higher skin friction resistance, which is the dominant component of total resistance. Because of this, the submerged model with $n f=1$ produced the lowest total resistance coefficient, whereas the model with highest $n f$ generated the highest magnitude.

Friction constituted the major component of resistance for the submerged model, specifically for models with a speed test of $10 \mathrm{~m} / \mathrm{s}$. For this reason, the friction coefficient defines the total resistance coefficient of the models.
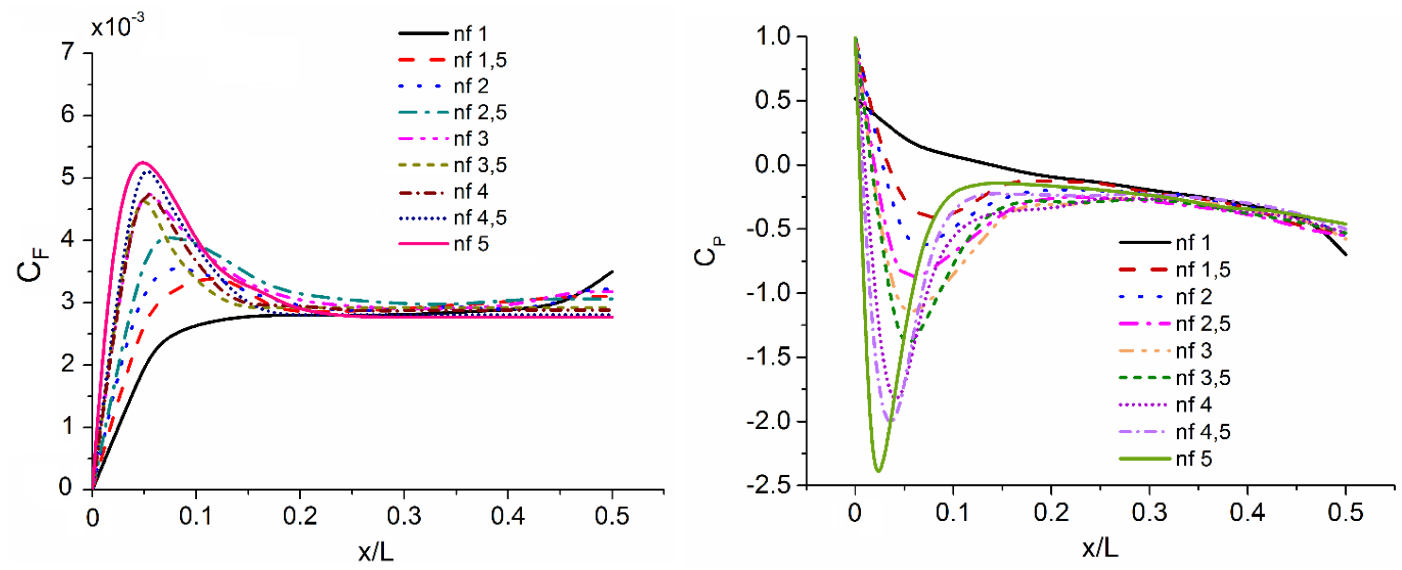

Figure 8 Friction coefficient distribution of the $n f$-based bow form models (left) and pressure coefficient distribution of the models (right) 
The friction coefficient distribution of the models is presented in Figure 8 (left). From the graph, the discrepancy in their distribution was induced by $n f$. Models with lower $n f$ had less fluctuating $C_{F}$ distribution after the fore end. This tendency was precipitated by the bow shape of the models, as well as the bow area. The $n f=1$ model had a low $C_{F}$ distribution trend because of its pointed conical shape, while the $n f=5$ model achieved the highest trend line because its stubbed foreend congested the flow, which also impacted the pressure distribution.

The pressure distribution on the models from the forebody to the midbody is shown in Figure 8 (right). The distinction in the trends was observed after the tip of the bow section since the difference in the bow form was based on the $n f$. Negative and positive accounts of $C_{P}$ depended on the fluid flows around it. Generally, the blunt shape (approaching $n f=5$ ) had a high negative distribution of $C_{P}$ after the bow tip, although conversely, the sharp form (approaching $n f=1$ ) had a positive distribution.

\subsection{Numerical Simulation of the Ellipse Models}

There are various parameters to determine the optimum bow shape for a submerged object: minimum resistance; minimum flow noise, specifically around acoustic sensors; and forebody arrangement. According to the results, the model with $n f=1$, or similar to the pointed shape, forms the least space for the bow arrangement, even though it produced the lowest resistance. However, the ellipse model is considered to be the more realizable form as it generated excellent resistance properties and had a proper available space in the forebody. Therefore, models with an $n f$ coefficient of between 2 and 3 and a ratio of 0.25 were included in the investigation.

Figure 9 (left) shows the total resistance coefficient of the models. The graph generated a similar common trend across the entire Reynold number range with a slight $n f$ variation. The models with a slightly larger $n f$ again produced moderately higher resistance.

Figure 9 (right) shows the pressure coefficient distribution of the ellipse models. The graph shows that higher $n f$ generated a higher pressure coefficient distribution. The $C_{P}$ and $C_{F}$ distribution impacted $C_{T}$; however, $C_{P}$ distribution shows no significant disparity between the ellipse models because the bow forms only differed slightly. For this reason, the total coefficient of the ellipse models resulted in a similar distinction.
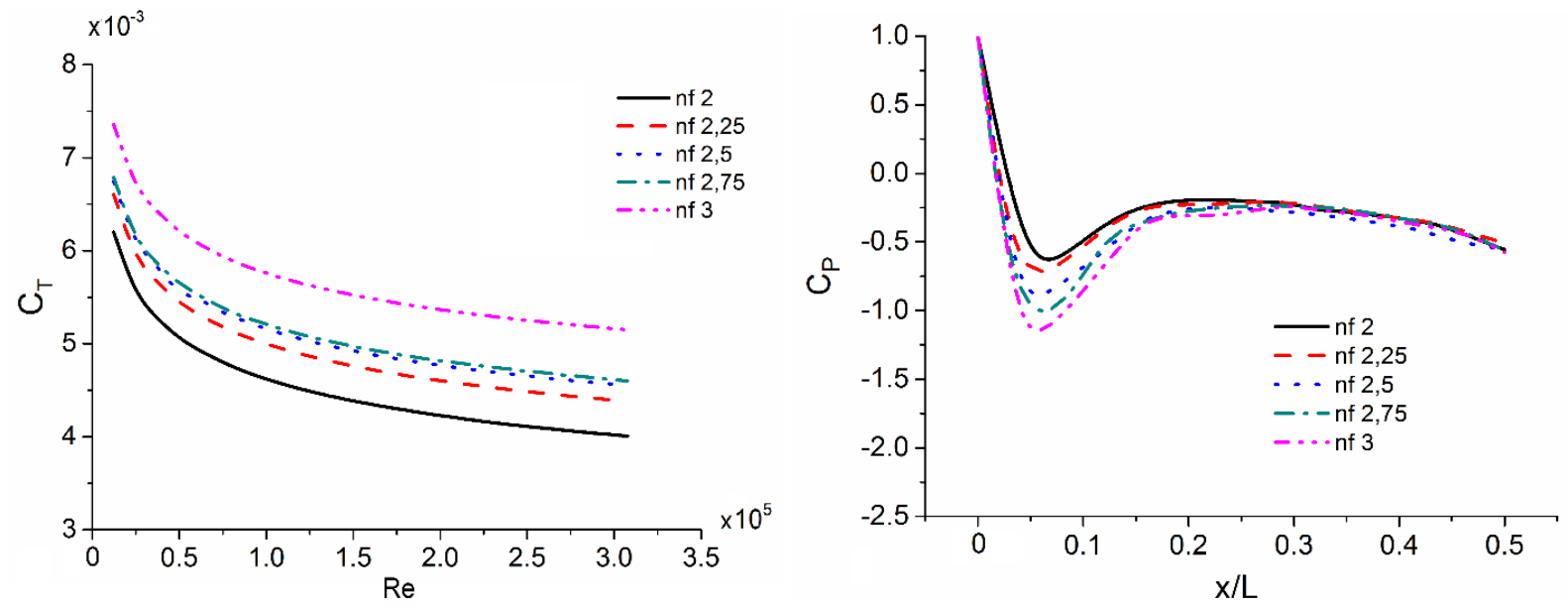

Figure 9 Total resistance coefficient of the ellipse models (left) and their pressure coefficient distribution (right)

The contour of the forebody determines the pressure difference. The pressure contour around the bow of the model with $n f$ is equal to 2, 2.5 and 3, as depicted in Figure 10. A dark filter was applied to the image in order to reveal the high-pressure difference in the flow; the shockwavelike phenomenon indicates this difference. 
The blunt model $(n f=3)$ formed a larger surface area with high pressure at the front-end than the other two models. After the shock (high pressure) on the tip, the flow accelerated from a point equivalent to one-fifth of the bow length. Because of its shape, the model with $n f=3$ developed a high accelerating flow, which led to very low pressure of around $-7 \times 10^{4} \mathrm{~Pa}$. This marked pressure difference led to flow separation, which might form cavitation from the low-pressure region of the accelerating flow and hence might emit high noise, which is undesirable.
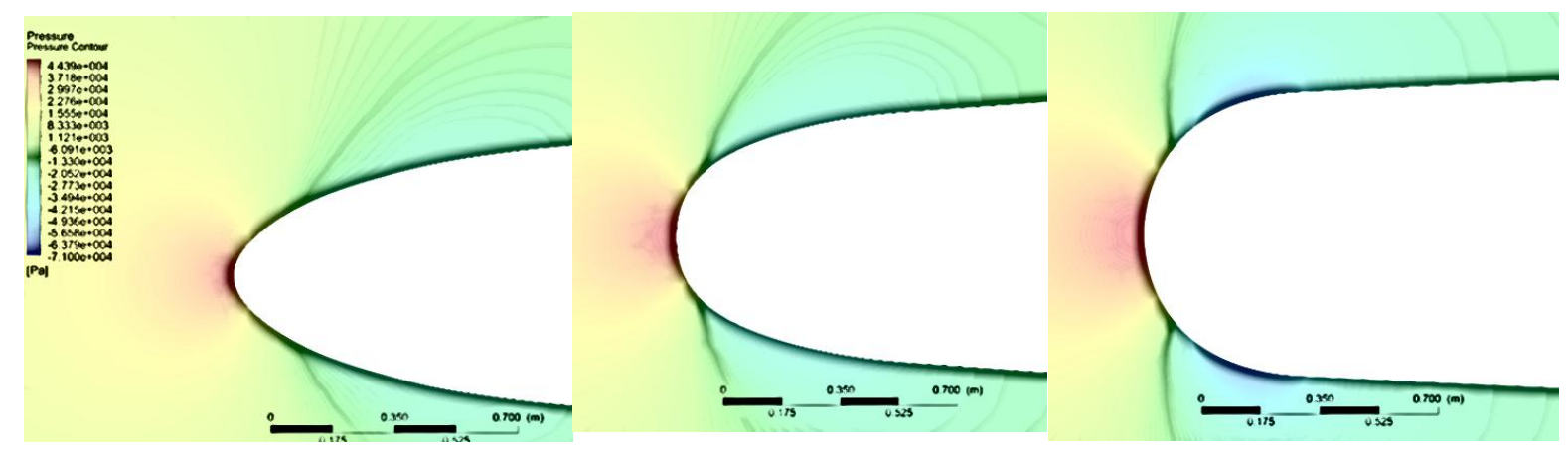

Figure 10 Pressure contour of model 8 with $n f=2$ (left), model 10 with $n f=2.5$ (centre), and model 12 with $n f=3$ (right)

An optimum bow design may be based on a bow shape that has a maximum area with minimum resistance. This study used a non-dimensional parameter designated as the bow efficiency coefficient $(\eta)$, written as:

$$
\eta=\frac{A_{\text {bow }}}{\operatorname{arc}_{\text {bow }}{ }^{2} C_{t}}
$$

where $A_{\text {bow }}$ is the bow area, and $\operatorname{arc}_{\text {bow }}$ is the arc length of the forebody. A considerable magnitude of this non-dimensional parameter indicates an optimum bow design.

A comparison of the bow efficiency coefficient $(\eta)$ of all models is shown in Figure 11. Models with an ellipse form or similar (from $n f=2$ to $n f=3$ ) generated a high bow efficiency coefficient compared to the other models. Therefore, according to the results, the submerged projectile model with $n f=2.75$ is the optimum form, as it has a sufficient bow area while maintaining less drag.

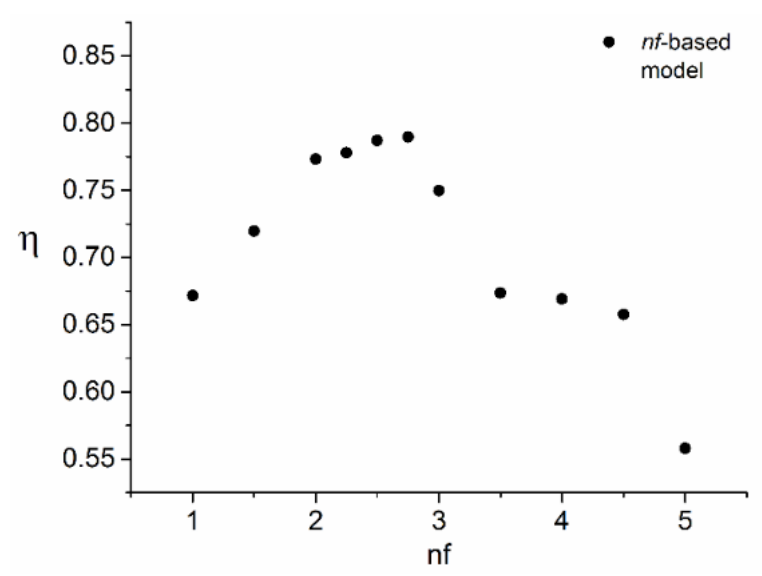

Figure 11 Comparison of the bow efficiency coefficient of all models 


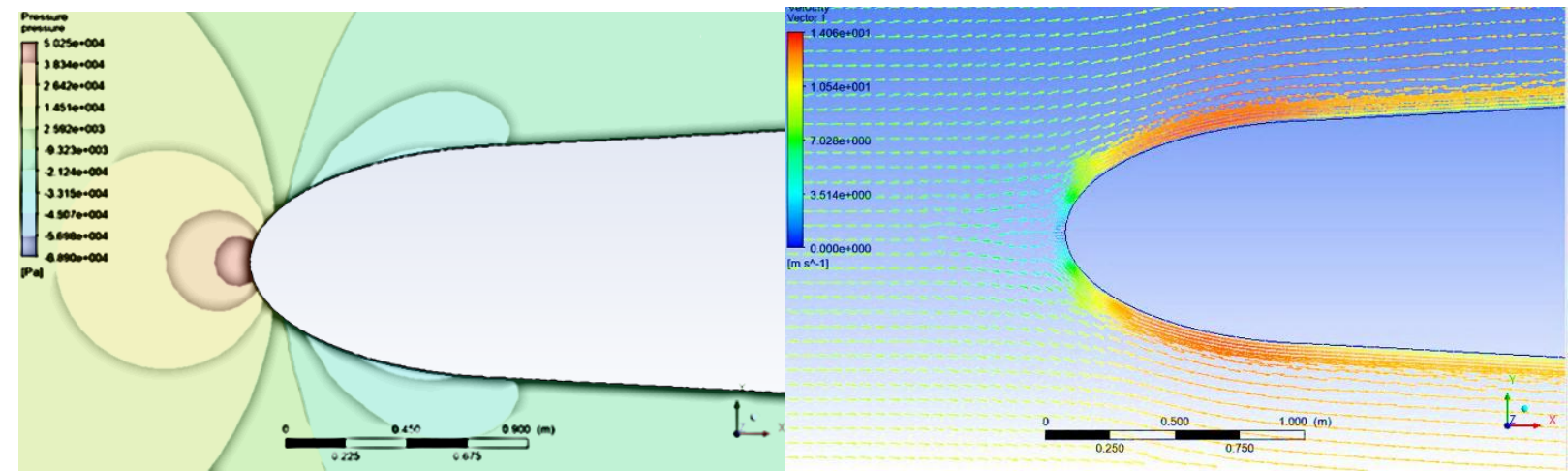

Figure 12 Pressure contour (left) and velocity vector (right) of model $11(n f=2.75)$

Figure 12 shows the pressure contour and fluid flow (velocity) vector around the forebody of the $n f=2.75$ model with a sheer scale. The contour influenced the pressure difference along the bow; slighter for a pointed bow and higher for a blunt bow shape by increasing the $n f$ coefficient, the pressure point on the tip of the forebody became extensive, causing high separation on the bow, which is undesirable.

In the velocity vector visualization, the color of the flow vector represents its velocity. Slight flow separation occurred from one-third of the forebody length from the front end. After the shock (high pressure) at the front end, the flow increased rapidly, thereby making the fluid pressure decrease, making the flow separate from the hull. Subsequently, the flow decreased, and the separation flow returned to the hull because of the regained fluid pressure after the increasing flow (low pressure); this flow is the optimum compared to the other forms.

\section{CONCLUSION}

The design of a submerged object depends on hydrodynamic aspects; the forebody is one of the critical concerns since flow of fluid toward the bow influences the remaining body. In this paper, CFD simulations of 2D submerged projectile models were conducted to examine this issue. The results provide a reasonable estimate of the resistance and flow characteristics of the models.

The model with $n f=1$ produced the lowest total drag since its wet surface area was smaller than that of the other models. However, the ellipse models attained better flow properties, and that with $n f=2.75$ was designated as the optimum design according to the bow efficiency coefficient. The pressure point on the front end became extensive for higher $n f$ models, thus making the pressure difference rise dramatically. On the ellipse models, the flow velocity increased from a point one-fifth along the bow length after the high pressure on the front end when the bow-head displaced the fluid. Subsequently, the rising velocity of the flow reduced the fluid pressure, thus creating a slight separation

The study has shown that a simplified CFD method using a commercial code could be a functional assessment of designing a submerged object at the initial stage. The optimum design based on the results of this study is suitable for projectile-shaped objects in deeply submerged conditions, such as submarines and torpedoes. Any consideration omitted from this paper may slightly revise the results.

Furthermore, involving additional analysis, such as the relation of the fluid around the bow to propulsion efficiency, would be considered worthy of examination, since the form of a hull affects the wake formation behind the self-propulsion object, as well as its resistance (Suastika et al., 2017). 


\section{ACKNOWLEDGEMENT}

This work is sponsored by the Ministry of Research, Technology, and Education of Republic Indonesia (NKB-1791/UN2.R3.1/HKP.05.00/2019).

\section{REFERENCES}

ANSYS, 2009. ANSYS FLUENT 12.0: Theory Guide, ANSYS, Inc.

Burcher, R., Rydill, L.J., 1994. Concepts in Submarine Design. Cambridge, UK: Cambridge University Press

Cao, L., Zhu, J., Zeng, G., 2016. Viscous-flow Calculations of Submarine Maneuvering Hydrodynamic Coefficients and Flow Field based on Same Grid Topology. Journal of Applied Fluid Mechanics, Volume 9(2), pp. 817-826

Chen, Y., Lu, C.-j., Cao, J-y., Chen, X., 2012. Application of Quadratic and Cubic Turbulence Models on Cavitating Flows around Submerged Objects. Theoretical \& Applied Mechanics, Volume 24(6), pp. 823-833

Farell, C., Guven, O., 1973. On The Experimental Determination of the Resistance Components of Submerged Spheroid. Journal of Ship Research, Volume 17(2), pp. 72-79

Fedor, R., 2009. Simulation of a Launch and Recovery of a UUV to a Submarine. Adelaide, Australia: Royal Institute of Technology KTH Marina System

Gross, A., Kremheller, A., Fasel, H., 2011. Simulation of Flow over Suboff Bare Hull Model. In: $49^{\text {th }}$ AIAA Aerospace Sciences Meeting including the New Horizons Forum and Aerospace Exposition, American Institute of Aeronautics and Astronautics

Jackson, H., 1992. Fundamental of Submarine Concept Design. SNAME, Volume 100, pp. 419448

Joubert, P.N., 2004. Some Aspects of Submarine Design. Part 1, Hydrodynamics. Fishermans Bend, Vic: DSTO Platforms Sciences Laboratory

Kim, D.-H., Park, W.-G., Jung, C.-M., 2012. Numerical Simulation of Cavitating Flow Past Axisymmetric Body. International Journal of Naval Architecture and Ocean Engineering, Volume 4(3), pp. 256-266

Kormilitsin, Y.N., Khalizev, O.A., 2001. Theory of Submarine Design. Riviera Maritime Media

Moonesun, M., Korol, Y.M., Nikrasov, V.A., Ursalov, A., Brajhko, A., 2016. CFD Analysis of the Bow Shapes of Submarines. Journal of Scientific and Engineering Research, Volume 3(1), pp. 1-16

Rattanasiri, P., Wilson, P.A., Phillips, A.B., 2015. Numerical Investigation of a Pair of Selfpropelled AUVs Operating in Tandem. Ocean Engineering, Volume 100, pp. 126-137

Renilson, M., 2015. Submarine Hydrodynamics. Lanceston, Australia: Springer

Saeidinezhad, A., Dehghan, A.A., Dehghan Manshadi, M., 2015. Experimental Investigation of Hydrodynamic Characteristics of a Submersible Vehicle Model with a Non-axisymmetric Nose in Pitch Maneuver. Ocean Engineering, Volume 100, pp. 26-34

Siswantara, A.I., Daryus, A., Darmawan, S., Gunadi, G.G.R., Camalia, R., 2016. CFD Analysis of Slurry Flow in an Anaerobic Digester. International Journal of Technology, Volume 7(2), pp. 197-203

Suastika, K., Nugraha, F., Utama, I.K.A.P., 2017. Parallel-middle-body and Stern-form Relative Significance in the Wake Formation of Single-screw Large Ships. International Journal of Technology, Volume 8(1), pp. 94-103

Suman, K., Rao, D.N., Das, H.N., Kiran, G.B., 2010. Hydrodynamic Performance Evaluation of an Ellipsoidal Nose for a High-speed Underwater Vehicle. Jordan Journal of Mechanical and Industrial Engineering, Volume 4(5), pp. 641-652

Toxopeus, S., 2008. Viscous Flow Calculation for Barehull DARPA SUBOFF submarine at Incidence. International Shipbuilding Progress, Volume 55(3), pp. 227-251 
Yang, C., Löhner, R., 2003. Prediction of Flows Over an Axisymmetric Body with Appendages. In: International Conference on Numerical Ship Hydrodynamics, Busan, Korea

Yanuar, Ibadurrahman, Waskito, K.T., Karim, S., Ichsan, M., 2017. Interference Resistance of Pentamaran Ship Model with Asymmetric Outrigger Configurations. Journal of Marine Science and Application, Volume 16(1). pp. 42-47 